\title{
Computer simulations of the dynamic properties of methane in a model silica gel
}

\author{
T.Patsahan*1 $^{*}$ A. Trokhymchuk ${ }^{1}$, M.Holovko ${ }^{1,2}$ \\ 1 Institute for Condensed Matter Physics \\ of the National Academy of Sciences of Ukraine, \\ 1 Svientsitskii Str., 79011 Lviv, Ukraine \\ 2 Ivan Franko Lviv National University, \\ 12 Dragomanov Str., 79005 Lviv, Ukraine
}

Received November 20, 2002

\begin{abstract}
Molecular dynamics (MD) simulations are reported for a Lennard-Jones fluid adsorbed into a model silica gel to study the dynamic properties of the adsorbed methane molecules. The mean-square displacement and velocity autocorrelation function of the adsorbed molecules are calculated for a set of supercritical temperatures at low (gas-like) and high (liquid-like) fluid densities and compared with the same data for a bulk fluid. The evaluated radial distribution functions illustrate the formation of a contact layer on the pore surface that is consistent with the decrease in the mobility of the adsorbed molecules in a porous environment. The calculated self-diffusion coefficient indicates a good quantitative agreement with the measured data for methane confined to the silica gel.
\end{abstract}

Key words: fluid, porous medium, diffusion, simulation, molecular dynamics.

PACS: $02.70 . N s, 45.20 . D d, 51.20 .+d, 61.43 . G t$

\section{Introduction}

The knowledge of the properties of the sorbate in specific porous solids is of great interest to both fundamental and applied science. Porous materials being extensively used as catalysts and adsorbents in the chemical industry appear to be a powerful stimulus for statistical-mechanics studies. There are two major features of the porous materials that affect the dynamics and other properties of the adsorbed fluids. These features are related to the structure of the porous confinement and to the degree of interaction of the sorbent molecules with the pore surfaces, i.e. they

*E-mail: tarpa@icmp.lviv.ua 
are related to wetting or non-wetting phenomena. The first one can be recognized as the geometrical aspect of the effect of the porous environment on the sorbate while the second one can be associated with the physical and/or chemical aspect of the adsorption process. A number of theoretical studies have been accomplished for the last two decades to model and investigate the physical adsorption into porous media. The researchers have primarily been concerned with highly idealized single pore spaces (slit-like, cylindrical and spherical pores).

Recently, the models for more complex porous microstructures that characterize silica Vycor glasses and silica gels have been developed [1,2] to study various properties of adsorbed fluids. These models are aimed at accounting for the effect of the structure and the topology of the pore space upon the fluid behavior in porous materials. The model of Vycor glass proposed by Rovere et al. [1] and utilized in a number of recent papers [3-5] is a single pore model which is quite acceptable due to the low porosity of this kind glasses where the interaction between fluid molecules from different pores can be neglected while the details of the pore structure can be taken into account. In particular case, considering the hydrophilic groups placed on the surface in porous silica-like Vycor glass, Rovere et al. [1] modelled the system on a site-site level that includes the interactions between the atoms of the adsorbed molecules and the pore material. Computer simulations based on such a model give quite realistic results for the structural and dynamic properties of the fluid adsorbed into the porous silica Vycor glass. However, in the case of silica gel we deal with a random porous structure. If for silica gel we use a model similar to that for silica glass [1], taking into account the randomness of pore volume distribution in the silica gels, the number of interaction sites will increase critically leading to an incredible increase of computing time. Therefore, one may prefer simplified models where the fluid-surface interaction is replaced with some effective potential.

A relatively simple but still nontrivial model for simple fluids adsorbed into a heterogeneous porous solid was formulated by Kaminsky and Monson (KM) [2]. Although KM model is simpler than the one used by Rovere et al. [1], it differs in principle from the previously studied models [6-8] for the adsorption of hard-spherelike fluids into hard-sphere porous media (hard-sphere matrix). The attractive fluidmatrix and fluid-fluid interactions are included in the KM model. Hence, the effect of temperature on the properties of an adsorbed fluid is presented. The fluid-matrix attraction is much stronger compared with the fluid-fluid interaction. Moreover, this model is also characterized by quite a large asymmetry of the diameters of matrix obstacles and of the adsorbed fluid molecules. The main attention in the studies based on the KM model has been paid so far to the structure and thermodynamic properties. To illustrate the utility of the KM model, the calculation of the Henry's law constant for methane adsorbed in a silica gel has been performed [2]. However, little attention has been paid to the fluid dynamics in heterogeneous microstructures. The asymmetry of energies and diameters may result in a strong accumulation of the fluid molecules in the vicinity of matrix particles. This has already been confirmed by Monte Carlo (MC) computer simulations [2,8]. Thus, we can expect that the mobility of fluid molecules could also be affected. 
The theoretical tools to investigate porous systems include the integral equation theory approaches [9-11] and computer simulation techniques [2,6-8]. Since in the present study our main goal is to investigate how the dynamic properties of the adsorbed fluid are altered under heterogeneous porous solid confinement, we applied molecular dynamics (MD) simulation technique. MD simulations are a versatile tool for a detailed description of the time evolution of the simulated system, simultaneously giving access to the evaluation of both structural and dynamic properties. In particular, we calculated the radial distribution functions, the running coordination numbers of fluid molecules around the matrix particles, mean square displacements, velocity autocorrelation functions and molecular self-diffusion in a porous medium trying to distinguish between the geometrical and interaction contributions. The remainder of the paper is organized as follows. The next section 2 describes the potentials of the fluid-fluid and the fluid-matrix interactions and the method used in the simulations. The results and the discussion are presented in section 3. Finally, the conclusions are drawn in section 4 .

\section{Modelling and computer simulations}

\subsection{Model description}

The bulk fluid is modelled by the truncated and shifted 12-6 Lennard-Jones potential:

$$
u_{\mathrm{ff}}(r)=\left\{\begin{array}{cc}
\phi_{\mathrm{LJ}}(r)-\phi_{\mathrm{LJ}}\left(R_{\mathrm{f}}\right), & r<R_{\mathrm{f}} \\
0, & r>R_{\mathrm{f}}
\end{array},\right.
$$

where $R_{\mathrm{f}}$ is the cut-off distance for the fluid-fluid interaction and

$$
\phi_{\mathrm{LJ}}(r)=4 \epsilon\left[\left(\frac{\sigma}{r}\right)^{12}-\left(\frac{\sigma}{r}\right)^{6}\right],
$$

with the parameters: $\epsilon / k=148.2 \mathrm{~K}, \sigma=0.3817 \mathrm{~nm}$ that correspond to the methanemethane interaction. The Lennard Jones diameter $\sigma$ is used as a unit of length scale through the whole paper. The bulk fluid is characterized by the reduced number density, $\rho_{\mathrm{f}}^{\star}=N_{\mathrm{f}} \sigma^{3} / V$, where $N_{\mathrm{f}}$ is the number of fluid molecules and $V$ is the volume occupied by the fluid system.

The model for a porous medium (or a porous matrix) is reduced to an equilibrium configuration of $N_{\mathrm{m}}$ large hard spheres (obstacles) of the diameter $D$. The ratio of the volume occupied by the matrix obstacles to the volume $V$ of the porous sample is $\eta=(\pi / 6) N_{\mathrm{m}} D^{3} / V$ and defines the porosity of the matrix, $1-\eta$. It has already been shown that this model is a reasonable first approximation to the structure of silica gel [12].

The essence of the modelling of the fluid adsorbed into a porous medium is the interaction between the fluid molecules and the matrix particles. In the present study this is based on the model developed by Kaminsky and Monson (KM) [2] for a methane adsorbed in a porous silica gel. According to the KM model an interaction 
between the fluid molecules and the spherical matrix obstacles has the following form:

$$
\phi_{\mathrm{KM}}(r)=\frac{2}{3} \pi \rho_{\mathrm{s}} D^{3} \epsilon_{\mathrm{s}}\left[\frac{\left(r^{6}+21 / 20 D^{2} r^{4}+3 / 16 D^{4} r^{2}+D^{6} / 192\right) d^{12}}{\left(r^{2}-D^{2} / 4\right)^{9}}-\frac{d^{6}}{\left(r^{2}-D^{2} / 4\right)^{3}}\right]
$$

with the parameters: $d=0.33 \mathrm{~nm}, \rho_{\mathrm{s}}=44 \mathrm{~nm}^{-3}, \epsilon_{\mathrm{s}} / k=339 \mathrm{~K}$ and $D=7.055 \sigma$. We employed this potential function to define two matrix models useful for the purposes of the present study. The first of them is equivalent to the KM model and we call it an attractive matrix due to the attractive nature of the fluid-matrix interaction:

$$
u_{\mathrm{fm}}^{\mathrm{ATT}}(r)=\left\{\begin{array}{cc}
\infty, & r<\frac{1}{2} D \\
\phi_{\mathrm{KM}}(r)-\phi_{\mathrm{KM}}\left(R_{\mathrm{m}}\right), & \frac{1}{2} D<r<R_{\mathrm{m}} . \\
0, & r>R_{\mathrm{m}}
\end{array}\right.
$$

Additionally, we introduce the repulsive matrix which is characterized by the repulsive potential of interaction between fluid molecules and matrix particles:

$$
u_{\mathrm{fm}}^{\mathrm{REP}}(r)=\left\{\begin{array}{cc}
\infty, & r<\frac{1}{2} D \\
\phi_{\mathrm{KM}}(r)-\phi_{\mathrm{KM}}\left(R_{\mathrm{m}}\right), & \frac{1}{2} D<r<R_{0} . \\
0, & r>R_{0}
\end{array} .\right.
$$

This last potential represents the repulsive part of the KM model interaction where the parameter $R_{0}=4.1526 \sigma$ was chosen in the way to ensure that the difference $\phi_{\mathrm{KM}}\left(r=R_{0}\right)-\phi_{\mathrm{KM}}\left(R_{\mathrm{m}}\right)$ is equal to zero.

The long-range attractive potential functions for the fluid-fluid and the fluidmatrix interactions, (1) and (4), both have been spherically truncated with the cut-off radii, $R_{\mathrm{f}}$ and $R_{\mathrm{m}}$, respectively. To eliminate the impulsive contribution to the force from the discontinuity of the potential, both truncated potentials have been shifted by the magnitude of the interaction energy at the cut-off distances. In the present study we used $R_{\mathrm{f}}=2.56 \sigma$ for the fluid-fluid interaction and $R_{\mathrm{m}}=12 \sigma$ for the fluid-matrix interaction.

\subsection{Molecular dynamics simulations}

The molecular dynamics (MD) simulations of the fluid adsorbed into a porous medium were performed through the adoption of commonly used velocity Verlet algorithm [13]. The modifications we introduced into this algorithm were caused by the presence in the simulation cell of the two quite distinct components, i.e. mobile fluid molecules and large static matrix particles. Thereby, we should solve the equations of motion exclusively for the fluid molecules, similarly to the case of the bulk fluid, but with the obstacles taken into account. For such a complex system, care has to be taken with respect to the total momentum which could not be equal 
to zero. To ensure the zero of the momentum in our algorithm, we monitored the total momentum of the system as a function of time and have observed only the negligible fluctuations around the zero value which did not effect the generated data. In general, the properties of the fluid in a porous environment are determined by the interaction within the fluid molecules, and by the interaction of the fluid molecules with the static obstacles. However, the role of a fluid-matrix interaction is frequently simplified and reduced to the effects such as geometrical obstructions, hydrodynamic drag, etc. To shed more light on the role of a fluid-matrix interaction and reveal the impact of geometrical and temperature effects on the behavior of a matrix fluid, four different systems (from A through D) have been considered and studied. The system A corresponds to the bulk fluid, i.e., $N_{\mathrm{f}}$ fluid molecules occupy a volume $V$. Each of the systems $\mathrm{B}$ and $\mathrm{C}$ is composed of the same (like system A) number of fluid molecules, $N_{\mathrm{f}}$. However, these molecules are infused into the two topologically identical porous media: one medium has an attractive fluid-matrix interaction (system B) while the second one has a repulsive fluid-matrix interaction (system C). Finally, the system D again is a bulk fluid (similar to the system A), but with an effective fluid density, i.e., the same $N_{\mathrm{f}}$ fluid molecules are placed into an effective volume, $V^{\prime}=V(1-\eta)$, which corresponds to the free volume in a porous medium in the systems B and C. The system D should be considered in order to show an effect of the presence of the matrix obstacles when the data for a matrix fluid (systems B and C) are compared against the bulk fluid (system A).

To choose the temperature region, we exploit the fact that in practice the adsorption into silica gels is usually observed at supercritical temperatures with respect to the bulk fluid. To ensure the single phase conditions, the reduced temperature, $T^{\star}=k T / \epsilon$, has been varied within the range from $T^{\star}=1.2$ to $T^{\star}=2.0$, which is above the bulk gas/liquid critical temperature $\left(T_{\mathrm{c}}^{\star} \sim 1.1\right)$ for the considered fluid model [15]. Hence, the temperature range $T^{\star}=1.2-2.0$ will be supercritical for the fluid adsorbed into a porous medium as well [16]. During the simulation runs, the average values of the desired temperatures were maintained using the velocity rescaling procedure according to the weak-coupling scheme due to Berendsen [14] with a coupling time constant $\tau^{*} \sim 0.1$ ps while the simulation time step $\Delta t$, was set to $2 \mathrm{ps}$. The production runs were started after the equilibration period of 440 ps.

All the matrix simulations (systems B and C) reported in the present study have been carried out for a fixed number of matrix particles, $N_{\mathrm{m}}=32$, which have been set randomly in the cubic box with the basic size $L=V^{1 / 3}$. This size is determined by the porosity of the matrix, $1-\eta$, which was fixed at the value corresponding to the KM model where $\eta=0.386$. The number of fluid molecules, $N_{\mathrm{f}}$, has been chosen to simulate the low (typical gas-like) and the high (typical liquidlike) fluid densities and was fixed at $N_{\mathrm{f}}=585$ (in some runs 456) and $N_{\mathrm{f}}=5385$ molecules, respectively. For each set of the fixed parameters of the adsorbed fluid, i.e. temperature and density, we carried out up to five different configurations of the matrix particles. The data that are reported have been obtained by the averaging over these configurations. 


\section{Results and discussion}

\subsection{Radial distribution functions}

Although our main goal is the dynamic properties, we begin with the discussion of the fluid-fluid and the fluid-matrix radial distribution functions (RDFs), $g_{\mathrm{ff}}(r)$ and $g_{\mathrm{fm}}(r)$, respectively. There were a few reasons that we persecuted by calculating and analyzing the RDFs. One of them was to verify the modified MD algorithm employed in the present study through the comparison of the obtained MD data against those computed by Vega et al. [8] from grand canonical Monte Carlo (GCMC) simulations. The second reason was to get conclusions about the effect of the porous environment on the fluid-fluid RDF through the comparison with the bulk data at the same temperature and density conditions. Further, we were curious about the local ordering of fluid molecules near the pore surfaces which could be revealed from the analysis of the fluid-matrix RDF. Finally, we intended to use the information on the structure ordering of the molecules of the adsorbed fluid while interpreting their dynamic properties.

The main results for the RDFs are summarized in figures $1-3$. The simulation data are presented for the two reduced temperatures, $T^{*}=1.2$ and 2.0, which correspond to bottom and top boundaries of the temperature range

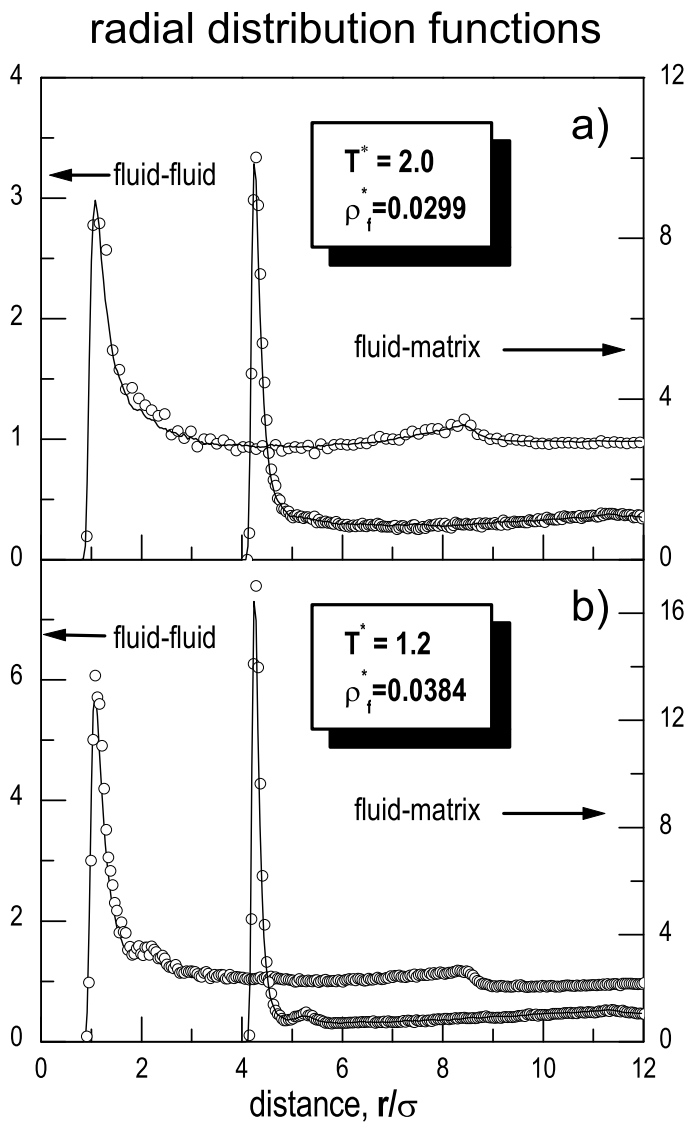

Figure 1. Attractive fluid-matrix interaction. Fluid-fluid and fluid-matrix radial distribution functions, $g_{\mathrm{ff}}(r)$ and $g_{\mathrm{fm}}(r)$, respectively, for a low fluid density at the temperatures $T^{*}=2.0$ (a) and 1.2 (b). Solid lines correspond to the data obtained from MD simulations of this work while circles are GCMC simulation data by Vega et al [8]. where the dynamic properties have been evaluated. Additionally, these simulation data show how the temperature affects the structural properties of the low density (gas-like) and the high density (liquid-like) fluids when they are adsorbed into a porous medium. First of all, for both densities (figures 1 and 2) we found an excellent agreement between MD data and those generated from GCMC by Vega et al. [8]. Small discrepancies should be attributed to the details of the truncation of potential functions. Analyzing the fluid-matrix RDFs for an attractive matrix, we observe the layered structure of the fluid molecules with 


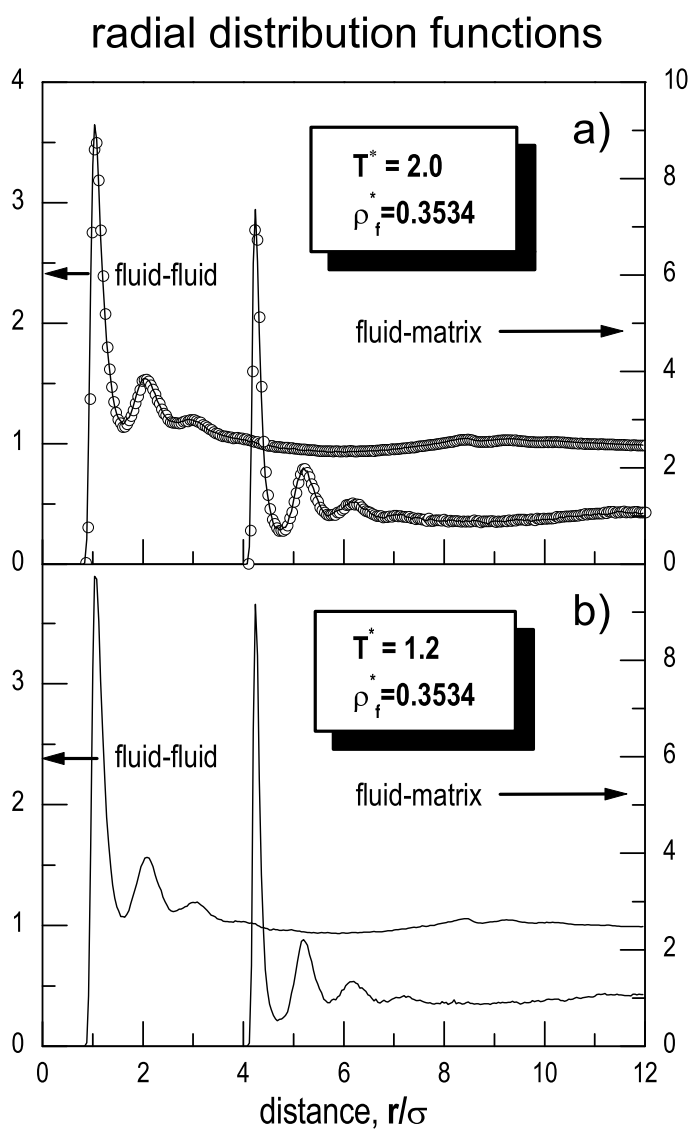

Figure 2. The same as in figure 1 but for a high fluid density. a well-defined contact layer around the matrix particles at both densities of the adsorbed fluid. When the density is fixed and the temperature is falling, the peaks of fluid-matrix RDFs increase as expected. However, at a fixed temperature, the same peaks decrease with the increase of the density of the matrix fluid, i.e. going from gas-like to liquidlike adsorbate. Opposite trends occur in the case of a repulsive matrix (figure 3 ) where the contact value of the fluidmatrix RDFs increases with the increase of the density, as expected. No layering of fluid molecules is observed for a gaslike adsorbate in the case of a repulsive matrix. To illustrate the formation of a contact layer, in figure 4 we present the snapshot of the MD configuration of the fluid molecules around the matrix particles for the case of an attractive matrix.

Using the fluid-matrix RDFs one can estimate the average numbers of the fluid molecules in a contact layer. To do this, we calculated the running coordination number,

$$
n(r)=4 \pi \rho_{\mathrm{f}}^{\star} \int_{0}^{r} g_{\mathrm{fm}}(x) x^{2} \mathrm{~d} x,
$$

for the fluid molecules adsorbed on the matrix particle. We can define the first coordination number, $n_{1}$, as the value of $n(r)$ at the distance $r$ that corresponds to the position of the first minima of $g_{\mathrm{fm}}(r)$. Corresponding data are collected in table 1 .

Table 1. The number of fluid molecules in the contact layer around the matrix particle

\begin{tabular}{ccccccc}
\hline \hline & & \multicolumn{2}{c}{ attractive matrix } & & \multicolumn{2}{c}{ repulsive matrix } \\
$T^{\star}$ & $\rho_{\mathrm{f}}^{\star}$ & $r_{\min }$ & $n_{1}$ & & $r_{\min }$ & $n_{1}$ \\
\hline 1.2 & 0.0384 & 4.95 & 34 & & 5.0 & 13 \\
& 0.3534 & 4.7 & 141 & & 4.8 & 115 \\
2.0 & 0.0299 & 5.15 & 23 & & - & - \\
& 0.3534 & 4.75 & 139 & & - & - \\
\hline \hline
\end{tabular}


One can see that the number of fluid molecules surrounding the matrix particles increases with the increase of the fluid density. The first coordination number has a very small dependence on the temperature for a high fluid density.

\subsection{Mean square displacement and velocity autocorrelation function}

The main subjects of our interest from the point of view of dynamic properties are two dynamic functions. The first is the time evolution of the mean square displacement (MSD), as defined by

$$
\left\langle r^{2}(t)\right\rangle=\frac{1}{N_{\mathrm{f}}}\left\langle\sum_{i}\left|\mathbf{r}_{i}(t)-\mathbf{r}_{i}(0)\right|^{2}\right\rangle,
$$

where the $\mathbf{r}_{i}(t)$ are the space coordinates of the center of mass of the fluid molecules at time $t$. The second dynamic function which we evaluated during simulation runs was the normalized velocity autocorrelation function (VACF),

$$
\varphi(t)=\frac{\sum_{i}\left\langle\mathbf{v}_{i}(t) \mathbf{v}_{i}(0)\right\rangle}{\sum_{i}\left\langle\mathbf{v}_{i}(0) \mathbf{v}_{i}(0)\right\rangle},
$$

with $\mathbf{v}_{i}(t)$ being the individual velocities of the fluid molecules at time $t$. The average $\langle\ldots\rangle$ is along the trajectory of each fluid molecule within the whole ensemble and over the time. The trajectory information can be employed to compute the time dependent diffusivity, $D(t)$. This can be evaluated either using the Green-Kubo expression [17],

$$
D(t)=\frac{1}{3} \int_{0}^{t} \varphi\left(t^{\prime}\right) \mathrm{d} t^{\prime}
$$

or the Einstein relation [17],

$$
D(t)=\frac{1}{6} \frac{\partial\left\langle r^{2}(t)\right\rangle}{\partial t} .
$$




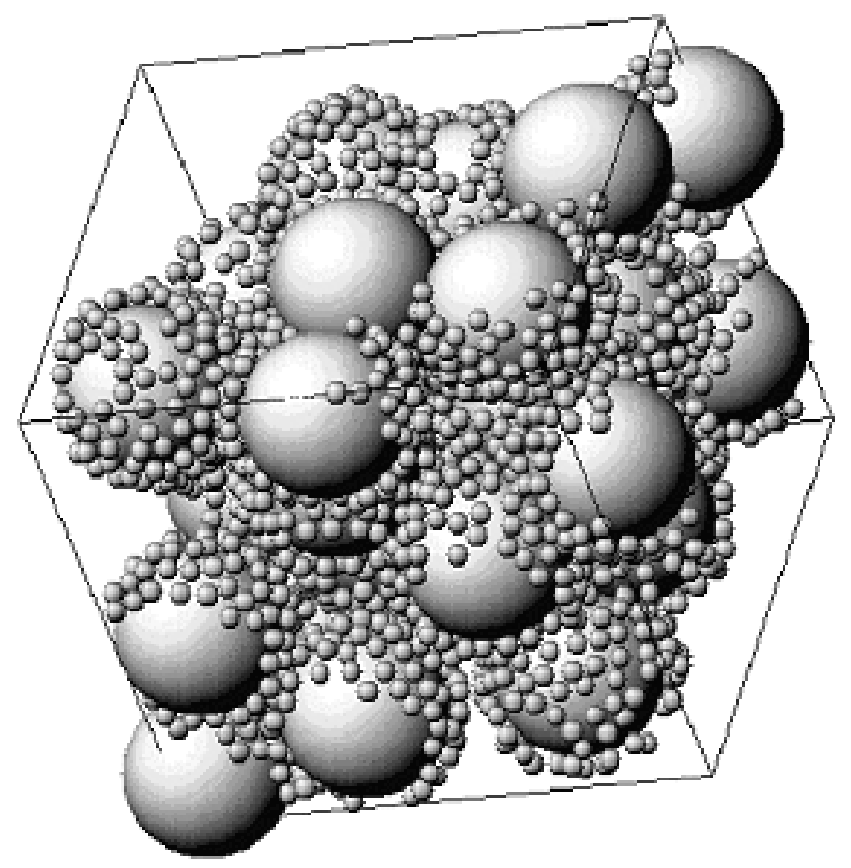

Figure 4. The snapshot of MD configuration of the fluid molecules around the matrix particles at a high fluid density, $\rho_{\mathrm{f}}^{\star}=0.3534$ and at temperature, $T^{*}=1.2$. Only fluid molecules belonging to the contact layers around the matrix particles are shown.

In general, we can summarize that the total length of the simulated trajectory was different for gas-like and for liquid-like fluids as well as it was different for bulk and for matrix fluids. Longer trajectories were needed in order to achieve a good statistical description of the MSD at a low density. At the fixed fluid density, final trajectories which correspond to the macroscopic self-diffusion coefficient $D=D(t \rightarrow \infty)$ are shorter when the fluid is adsorbed in a porous medium. Relatively shorter trajectories (comparatively to MSD) are needed for recording VACF to ensure their decay to the final value of zero (within the statistical accuracy of the simulation data). Generally speaking, the random structure of a porous medium may lead to the anisotropy of the diffusion process. However, we were interested in the average features of the molecule dynamics only.

The set of MSDs as a function of the observation time for the low and the high fluid densities are shown in figures $5 \mathrm{a}$ and $5 \mathrm{~b}$, respectively. Each figure contains two groups of curves which correspond to the bulk fluid (dashed lines) and to the matrix fluid (solid lines) under the same temperature conditions. As it is already known from the bulk studies, the molecules have a much bigger displacement for the low fluid density than for the high fluid density. The MSD is also bigger at a higher temperature. The presence of the matrix particles leads to the slowing down of the diffusion motion: fluid molecules diffuse at considerably shorter distances in the porous medium than in the bulk conditions. This is observed for both densities and for all temperatures studied. 

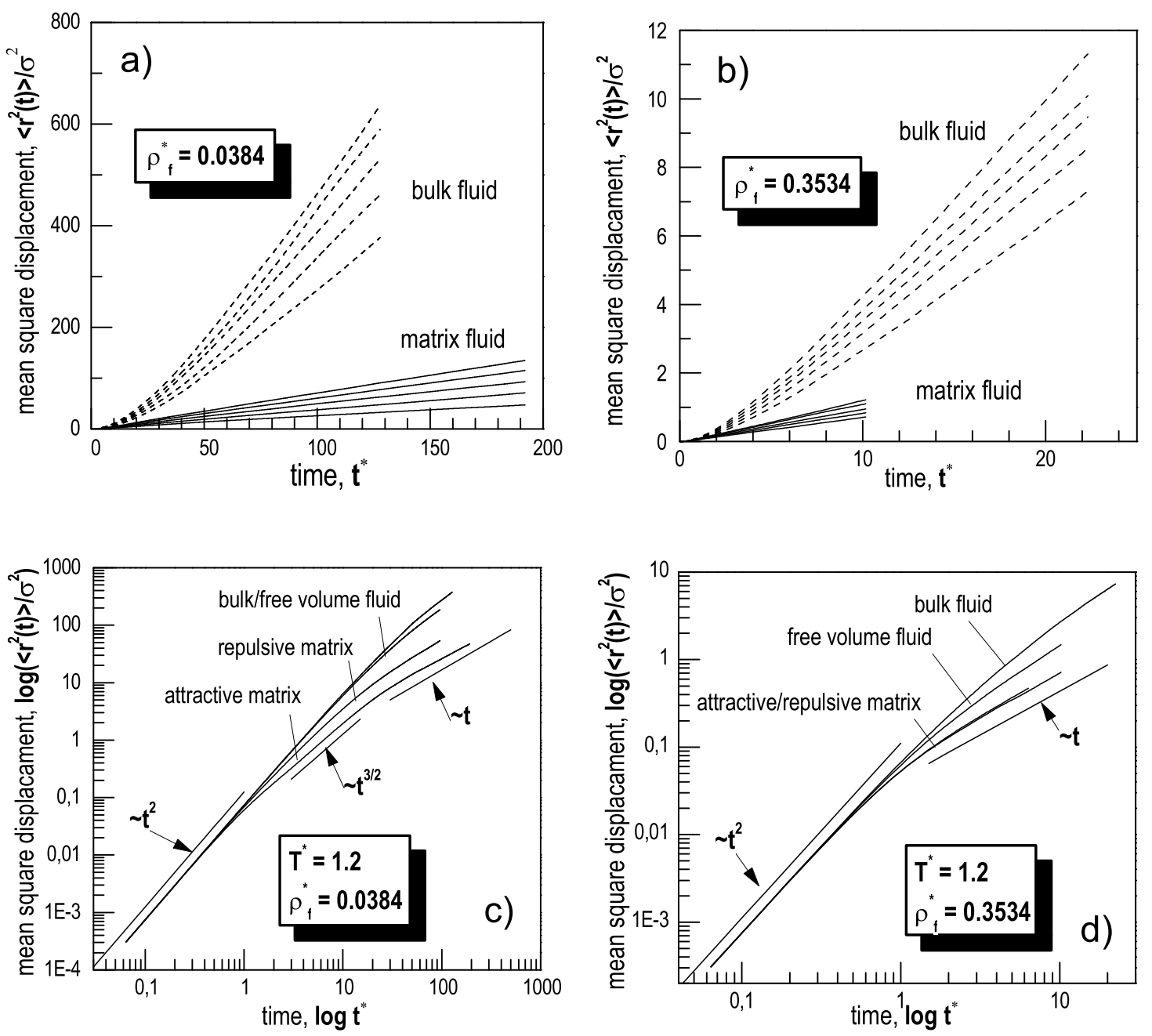

Figure 5. MD simulation data for the mean square displacement. (a): Comparison of the bulk and matrix fluids at different temperatures, $T^{*}=1.2 ; 1.4 ; 1.6 ; 1.8 ; 2.0$ (from the bottom to the top) and a for low fluid density; (b): The same as in (a) but for a high fluid density; (c): Comparison of the bulk fluid, free volume fluid and matrix fluid with attractive and repulsive fluid-matrix interactions at temperature $T^{*}=1.2$ for a low fluid density; (d): The same as in (c) but for a high fluid density. 
The time dependence of the MSD is different at different periods of time. Three different time regimes have been distinguished by analyzing the movement of the tracer from the time dependence of the correlation function of the scattered-light intensity [18,19]: (i) the diffusion process is normal at short times, (ii) becomes anomalous at moderate times and (iii) returns to normal for large delay times. Normal diffusion assumes here that time dependence of the fluid molecule dynamics is subject to the law of deterministic motion (Newtonian dynamics) on the initial ballistic phase and satisfies the Fick's second law and isotropy (Euclidean dynamics) on a long time scale. The analysis of the simulation data on this subject is presented in figures $5 \mathrm{c}$ and $5 \mathrm{~d}$ for the low and the high fluid densities, respectively. In order to extract the information from the simulation data, the log-log plots of MSD are used.

Two linear regions (at the initial and final stage) can be easily recognized for each set of the data presented on the plots in figures $5 \mathrm{c}$ and $5 \mathrm{~d}$. In general, every linear region on logarithmic scale indicates a distinct diffusive regime, described by a power law behavior $D(t) \sim t^{\alpha}$ where the power $\alpha$ can be determined from the slope of the straight line fitting the data. Below the time, $t^{\star}=t\left(\epsilon / m \sigma^{2}\right)^{1 / 2} \sim 1$, the straight line has the slope $\alpha=2$, corresponding to Newtonian dynamics, i.e. $\left\langle r^{2}(t)\right\rangle=v^{2}(0) t^{2}$. Well above $t^{\star} \sim 1$, the straight line has the slope $\alpha=1$ corresponding to Einsteinian regime, i.e. $\left\langle r^{2}(t)\right\rangle \sim D t$. In the case of a bulk fluid, these two straight lines intersect, defining the characteristic distance $\lambda$ and time $\tau$ that separate the two diffusive regimes. Between these two regimes, the displacement profile is not linear, representing the transition from deterministic (quasi-free) molecule motion to the Einsteinian diffusion (molecule motion is affected by the collisions with the other molecules). Comparing the MSD curves for the bulk fluid with the MSD curves for the gas-like matrix fluid (figure 5c) we see that, indeed, as it was found experimentally in the studies of probe diffusion through polyacrylamide gels [18], the shape of the MSD profile has an additional linear region in between two normal diffusion regimes. The slope of this linear region for a low density adsorbate is about $\alpha=3 / 2$ indicating a perturbation of the molecule trajectory due to the collision with the obstacles. This behavior only quantitatively depends on the nature of the surface of the matrix particles (attractive or repulsive fluid-matrix interaction). For an attractive matrix, the transition time from ballistic behavior to anomalous diffusion is shorter as well as the crossover from anomalous diffusion to the normal diffusion over long distances is more delayed. After entering the anomalous diffusion regime, MSD is permanently larger for repulsive obstacles.

Anomalous diffusion is strongly affected by the density of the adsorbed fluid (figure 5d). In particular, the linear region at moderate times practically does not exist for the liquid-like adsorbate, i.e. the molecules are found to follow the normal (Brownian) diffusion directly after the initial ballistic phase. As in the case of a low fluid density, time dependence of the MSD is the same for both attractive and repulsive matrix particles. The explanation to this behavior is that at a high density of the adsorbed fluid, the surface coverage of matrix particles is very high for both (repulsive and attractive) matrices (see the first coordination numbers in table 1) and 

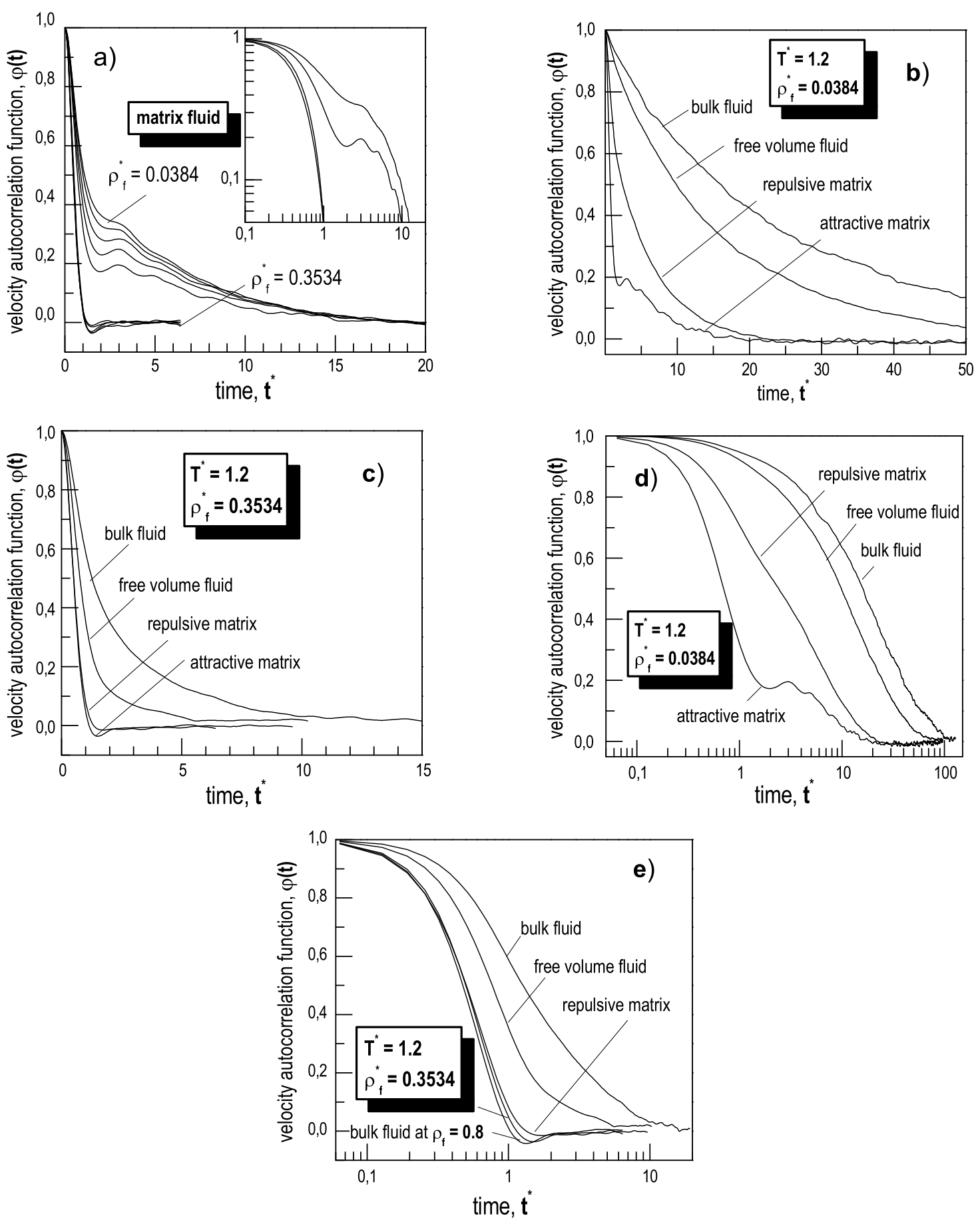

Figure 6. MD simulation data for normalized velocity autocorrelation function. (a): Comparison between the matrix fluids at low and high fluid densities and at different temperatures, $T^{*}=1.2 ; 1.4 ; 1.6 ; 1.8 ; 2.0$ (from the bottom to the top); (b): Comparison of the bulk fluid, free volume fluid and matrix fluid with attractive and repulsive fluid-matrix interactions at temperature $T^{*}=1.2$ for a low fluid density; (c): The same as in (b) but for a high fluid density; (d): The same as in (b) but in log scale; (e): The same as in (c) but in log scale. 
the tracer cannot "see" the naked surface of the matrix obstacles but can "see" only the fluid molecules from the adsorbed (or contact) layer. Due to this, the diffusion process in the porous medium at a high density of the adsorbed fluid is qualitatively similar to the diffusion of the bulk (free volume or effective) fluid at a higher density.

A noticeable difference in the dynamic properties of gas-like and liquid-like matrix fluids is also evidenced by the data simulated for the VACFs which are shown in figure 6. For each fluid density the set of five curves are displayed (see figure 6a) to illustrate the temperature effect. The both groups of VACFs relax following the qualitatively different patterns, which means that the motions of individual molecules are essentially different. It is notable that $\varphi(t)$ relaxes much faster in the case of a high density adsorbate and assumes the negative values at $t^{\star} \sim 1$. The slow relaxation at a low fluid density is superimposed by a weak non-periodic oscillating pattern. The normalized VACFs, evaluated for bulk, for free volume, and for both (attractive and repulsive) matrix fluids are shown in figures $6 \mathrm{~b}-6 \mathrm{e}$ for both the gas-like and the liquid-like fluids. One can see, that $\varphi(t)$ of the matrix fluids (both repulsive and attractive) decays faster than that in the bulk fluid, regardless of time. The deviation is observed starting from the times $t^{\star} \sim 0.2$ and appears to be significant at the times greater than the time $t_{\text {con }}^{\star}$ roughly required for the molecules to diffuse into contact with the matrix particles. In contrast to the bulk fluid, the well defined minima in $\varphi(t)$ profile for the case of a matrix fluid are seen at short times.

\subsection{Macroscopic self-diffusion coefficient}

Both expressions, equations (9) and (10), can be used to predict the macroscopic self-diffusion coefficient, $D=D(t \rightarrow \infty)$. However, in both cases the functions $\varphi(t)$ and $\left\langle r^{2}(t)\right\rangle$ have to be known at the large times compared to the characteristic time of correlations between the tagged molecule and its immediate neighbors. At these times, which determine the hydrodynamic region, we can expect that $\left\langle r^{2}\right\rangle$ behaves like a linear function of $t$. Hence, $D$ can be calculated as the limiting slope of the MSD profile. In such a context an evaluation of the self-diffusion coefficient, $D$, through the VACF is more rigorous. This is illustrated in figure 7 where the MD simulation data are displayed for the time-dependent self-diffusion, $D(t)$, evaluated from both the VACFs and the MSDs and for all four systems discussed in the present study. One can see that for the bulk as well as for the matrix fluids both roots lead to consistent results (within each simulated system) at large times. In the bulk case, both procedures give consistent results with a similar uncertainty which is of the order of $5 \%$ [20]. The same seems to be valid for the matrix fluid: the final values of self-diffusion as $t \rightarrow \infty$ are equal being calculated from MSD or VACF. However, these final values are different for each simulated system; the difference between attractive and repulsive matrix vanishes for a high fluid density. An interesting observation that follows from the data shown in figure 7 concerns the maxima for self-diffusion versus time in the case of both matrix fluids that is clearly seen when the log scale is used (figures $7 \mathrm{c}$ and $7 \mathrm{~d}$ ). This is an important qualitative difference between the bulk and the matrix fluids and can serve as another evidence of the possible anomalies in the diffusion processes in a porous environment. 

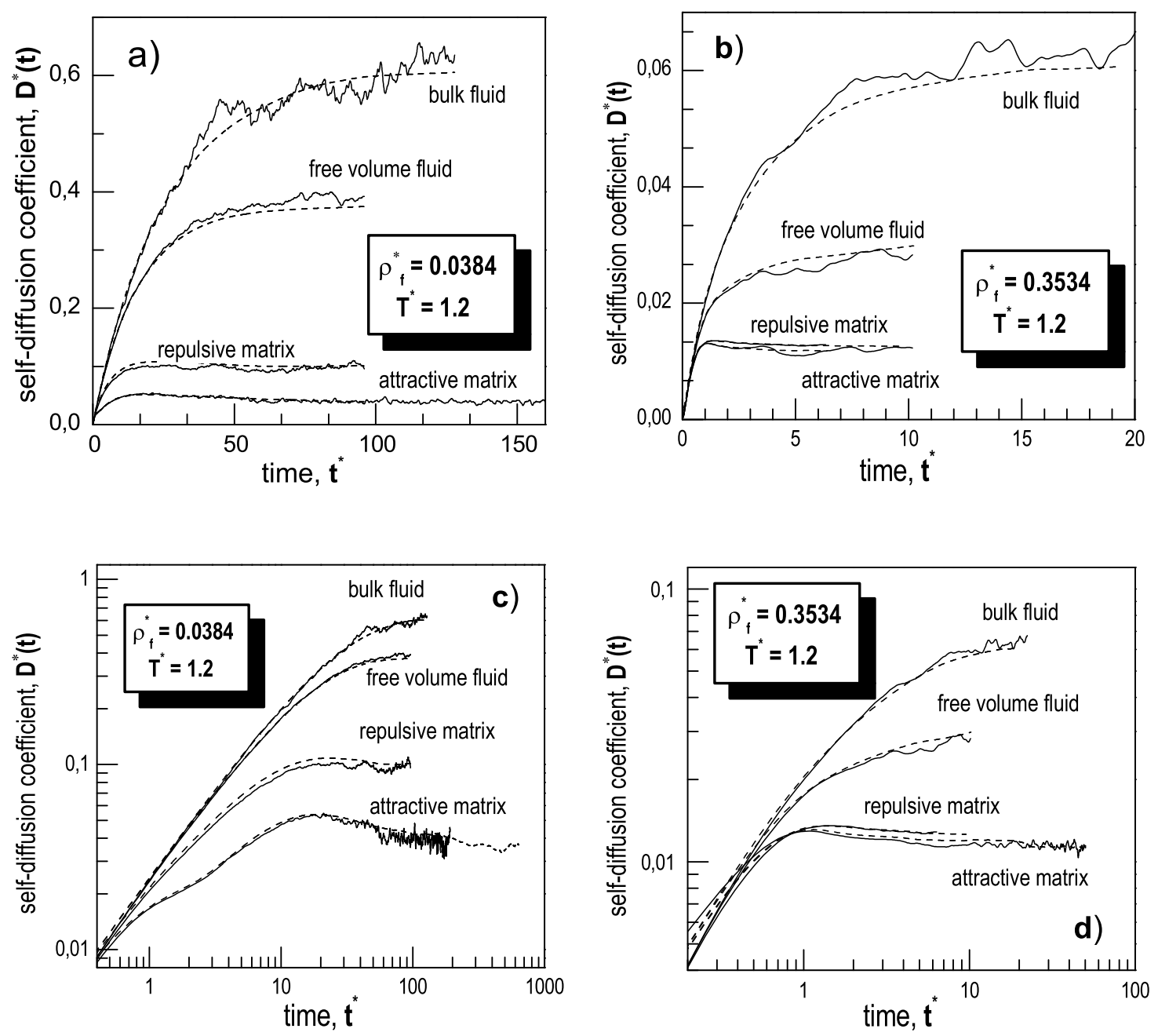

Figure 7. Time-dependent self-diffusion at temperature $T^{*}=1.2$. Solid lines correspond to the results obtained from the MSD data while dashed lines correspond to the results evaluated from the VACF data. (a): Comparison of the bulk fluid, free volume fluid and matrix fluids with attractive and repulsive fluid-matrix interactions for low fluid density; (b): The same as in (a) but for a high fluid density; (c): The same as in (a) but in log scale; (d): The same as in (b) but in $\log$ scale. 


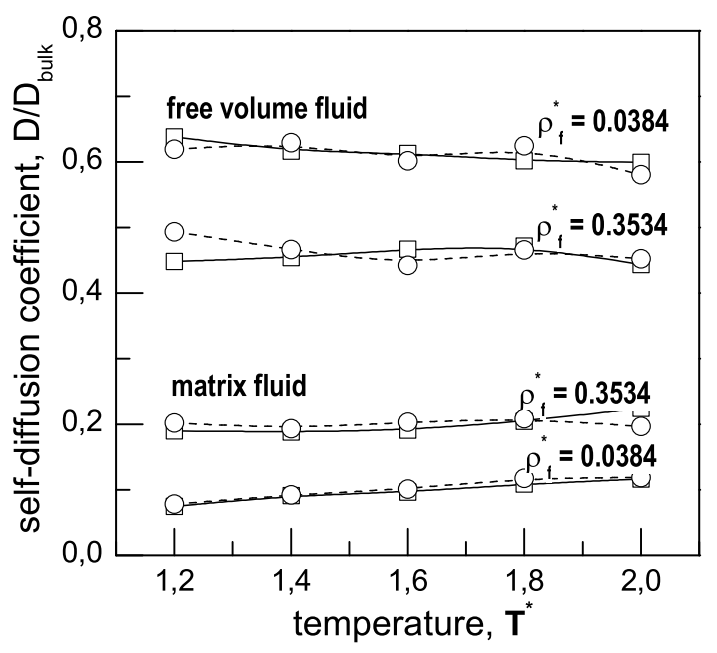

Figure 8. Relative self-diffusion coefficient of the attractive matrix $\left[D_{\text {matr }} / D_{\text {bulk }}\right]$ and the effective (free volume) $\left[D_{\text {eff }} / D_{\text {bulk }}\right]$ fluids versus temperature $T^{*}$, for the low and the high fluid densities. The meaning of solid and dashed lines is the same as in figure 7 .

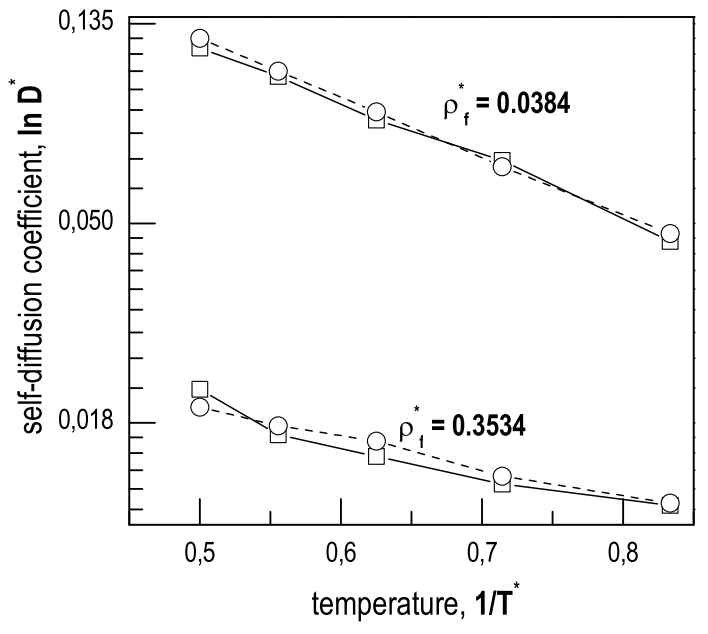

Figure 9. Logarithmic self-diffusion coefficient of the attractive matrix fluid versus $1 / T^{*}$ for the low and the high fluid densities. The meaning of solid and dashed lines is the same as in figure 7 .

The simulation data for the macroscopic self-diffusion coefficient, $D$, are summarized in figures 8-10. Two sets of data for each temperature and density case correspond to the self-diffusion coefficient obtained from the MSDs (solid lines) and from the VACFs (dashed lines). We see, that in all cases the agreement between both procedures is quite reasonable. The ratios of the diffusion coefficients of the attractive matrix fluid (system B) and the bulk fluid with an effective density (system D) to the corresponding quantity for the bulk conditions (system A), $D_{\text {matr }} / D_{\text {bulk }}$ and $D_{\text {eff }} / D_{\text {bulk }}$, respectively, clearly show that $D_{\text {matr }}$ changes relatively to both the bulk fluid and the bulk fluid with an effective density (figure 8). This means, that the diffusion processes in a porous environment are significantly affected not only by the decrease of the volume but also by the presence of the matrix obstacles; diffusion is considerably slower in this particular case of a porous medium. This effect is more pronounced for the gas-like adsorbate where $D_{\text {matr }}$ is one order of magnitude lower than for the free volume case, $D_{\text {eff }}$, while for a high density of the adsorbed fluid one can see a half of the order of magnitude decrease. Examining these data, we see that most of the decrease in $D_{\text {matr }}$ is caused by the presence of a porous confinement. The substitution of the matrix fluid by the bulk fluid with the effective density that, indeed, is associated with the decrease of diffusion, does not correspond and is not equivalent to the effect of a porous confinement. Not only the magnitudes of the diffusion coefficients $D_{\text {eff }}$ and $D_{\text {matr }}$ are different ( $D_{\text {matr }}$ is always lower than $D_{\text {eff }}$ at the same fluid density and temperature), but the dependence on temperature and density in both cases is qualitatively different: ratio $D_{\text {matr }} / D_{\text {bulk }}$ increases with 


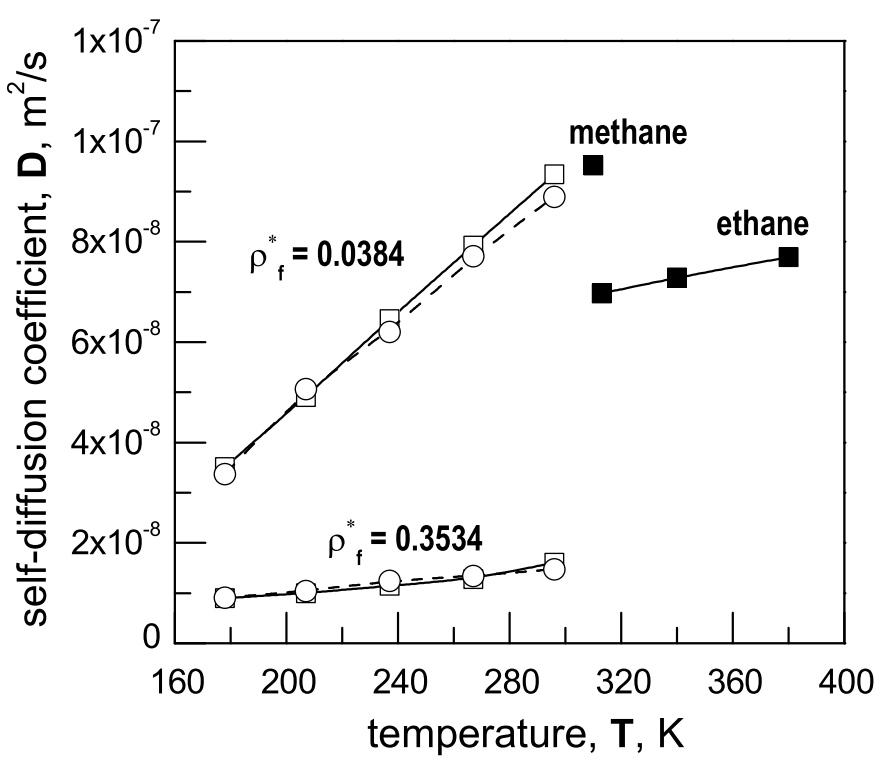

Figure 10. Self-diffusion coefficient of the adsorbed methane molecules in the silica gel versus temperature $T$ in real units for gas-like and liquid-like fluid densities. Square symbols with a solid line correspond to the results obtained from the MSD data, while circles with a dashed line correspond to the results evaluated from the VACF data. The filled symbols correspond to the experimental data for methane and ethane gases in silica gel.

the rise of temperature while $D_{\text {eff }} / D_{\text {bulk }}$ decreases; ratio $D_{\text {matr }} / D_{\text {bulk }}$ for a low fluid density is smaller than for a higher fluid density while $D_{\text {eff }} / D_{\text {bulk }}$ has an opposite trend.

The effect of temperature on the diffusivity can be seen in figure 9. This plot shows that the diffusion of gas-like and liquid-like adsorbates in a porous medium is an activated process, i.e. it follows the Arrhenius equation,

$$
D(T)=D_{0} \exp \left(-E_{\mathrm{a}} / R T\right)
$$

where $D_{0}$ is called the preexponential factor, $E_{\mathrm{a}}$ is the activation energy, $R$ is the gas constant, and $T$ is the temperature. The estimate of an activation energy is $3.53 \mathrm{~kJ} / \mathrm{mol}$ for low a density of fluid molecules and $1.80 \mathrm{~kJ} / \mathrm{mol}$ for the fluid molecules at a liquid-like fluid density.

The absolute values of the prediction for the self-diffusion coefficient for the methane adsorbed in silica gel are shown in figure 10. From this plot one can also see the dependence of the self-diffusion coefficient $D_{\text {matr }}$ on the temperature: with the rise of the temperature, the diffusion increases and is accelerated at a low fluid density relative to high fluid density. Our estimates, $D_{\text {matr }}=0.875 \cdot 10^{-7} \mathrm{~m}^{2} / \mathrm{s}$, for the gas-like fluid density at $T=296 \mathrm{~K}$ are in a reasonable agreement with the experimental value $0.952 \cdot 10^{-7} \mathrm{~m}^{2} / \mathrm{s}$ for the pore diffusivity of the methane gas in silica gel at $T=310 \mathrm{~K}[21]$. 


\section{Conclusions}

Summarizing, the MD simulations are applied while investigating the dynamic properties of simple fluids adsorbed in a porous medium. The study is based on the model for the adsorption of methane in silica gel developed by Kaminsky and Monson [2]. MD simulations that we have performed allow us to examine the dependence of the dynamic properties of the adsorbed fluid on: (i) the presence of static obstacles that form a random porous structure; (ii) specific (attractive or repulsive) interaction between the fluid molecules and the matrix particles; (iii) the density of the adsorbed fluid; (iv) the temperature conditions in the system.

To analyze the dynamic properties, both the velocity autocorrelation functions and the mean square displacements have been evaluated. Comparing the MSD curves for the bulk fluid with the MSD curves for the matrix fluid we found that the shape of the MSD profile has an additional linear region in between two normal diffusion regimes. The slope of this linear region for a low density adsorbate is about $\alpha=3 / 2$ indicating a perturbation of the molecule trajectory due to collision with the obstacles. This behavior qualitatively agrees with experimental findings in the studies of probe diffusion through the polyacrylamide gels [18]. Such an anomaly in the MSD versus time is not practically seen for a high density of the matrix fluid since the surface coverage of the matrix particles by the fluid molecules is very high and the tracer cannot "see" the naked surface of the matrix obstacles but it can "see" only the fluid molecules from the adsorbed layer. Due to this, the diffusion process in a porous medium at a high density of the adsorbed fluid is qualitatively similar to the diffusion of the bulk fluid at a higher density.

Both the velocity autocorrelation functions and the mean square displacements have been used to predict the macroscopic self-diffusion coefficient, $D$. It was noticed that the self-diffusion coefficient is more convenient to be obtained from the velocity autocorrelation function. The presence of the matrix particles affect the mobility of fluid molecules. At a low density of the matrix fluid, $D$ strongly depends on the temperature. The effect of temperature decreases when the density of the adsorbed fluid increases. Moreover, we have shown that the diffusion of both gas-like and liquid-like adsorbates in a porous medium is an activated process, i.e. it follows the Arrhenius equation. The self-diffusion coefficient is smaller for a matrix fluid than for a bulk fluid. It is shown, that $D$ decreases not only due to the decrease of the space available (an excluded volume effect) for adsorption but also due to the specific geometry of the confinement. Comparison of the calculated self-diffusion coefficient with the measured data for the methane confined to the silica gel indicates a good quantitative agreement.

\section{References}

1. Rovere M., Ricci M.A., Vellati D., Bruni F. // J. Chem. Phys., 1998, vol. 108, p. 9859.

2. Kaminsky R.D., Monson P.A. // J. Chem. Phys., 1991, vol. 95, p. 2936.

3. Spohr E., Hartnig C., Gallo P., M. Rovere // J. Mol Liq., 1999, vol. 80, p. 165. 
4. Gallo P., Rovere M., Ricci M.A., Hartnig C., Spohr E. // Europhys. Lett., 2000, vol. 49, p. 183.

5. Gallo P., Ricci M.A., Rovere M. // J. Chem. Phys., 2002, vol. 116, p. 342.

6. Fanti L.A., Glandt E.D., Madden W.G. // J. Chem. Phys., 1990, vol. 93, p. 5945.

7. Lomba E., Given J.A., Stell G., Weis J.J., Levesque D. // Phys. Rev. E, 1993, vol. 48, p. 233.

8. Vega C., Kaminsky R.D., Monson P.A. // J. Chem. Phys., 1993, vol. 99, p. 3003.

9. Madden W.G., Glandt E.D. // J. Stat. Phys., 1988, vol. 51, p. 537.

10. Given J.A., Stell G. // J. Chem. Phys., 1992, vol. 97, p. 4573.

11. Trokhymchuk A., Pizio O., Holovko M., Sokolowski S. // J. Chem. Phys., 1997, vol. 106, p. 200.

12. MacElroy J.D., Raghavan K. // J. Chem. Phys., 1990, vol. 93, p. 2068.

13. Allen M.P., Tildesley D.J. Computer Simulation of Liquids. Oxford, Oxford Science Publications, 1987.

14. Berendsen H.J.C., Postma J.P.M., van Gunsteren W.F., Di Nola A., Haak J.R. // J. Chem. Phys., 1984, vol. 81, p. 3684.

15. Trokhymchuk A., Alejandre J. // J. Chem. Phys., 1999, vol. 111, p. 8510.

16. Trokhymchuk A., Sokolowski S. // J. Chem. Phys. 1998, vol. 109, p. 5044.

17. Hansen J.P., McDonald I.R. Theory of Simple Liquids. London, Academic Press Inc., 1976.

18. Suzuki Y., Nishio I. // Phys. Rev. B, 1992, vol. 45, p. 4614.

19. Netz P.A., Dorfmüller T. // J. Chem. Phys., 1995, vol. 103, p. 9074.

20. Levesque D., Verlet L. // Phys. Rev. A, 1970, vol. 2, p. 2514.

21. Gangwal S.K, Hadgins R.R., Silveston P.L // The Canadian J. of Chem. Engineering, 1979, vol. 57, p. 609. 


\title{
Комп'ютерне моделювання властивостей метану у модельному силікагелі
}

\author{
Т.Пацаган ${ }^{1}$, А.Трохимчук ${ }^{1}$, М.Головко ${ }^{1,2}$ \\ 1 Інститут фізики конденсованих систем НАН України, \\ 79011 Львів, вул. Свєнціцького, 1 \\ 2 Національний університет ім. Івана Франка, \\ 79005 Львів, вул. Драгоманова, 12
}

Отримано 20 листопада 2002 р.

\begin{abstract}
Було здійснено комп'ютерне моделювання методом молекулярної динаміки (МД) для Ленарда-Джонсівського флюїда адсорбованого в модельному силікагелі. Обчислено функції середньо-квадратичного відхилення координат та автокореляційні функції швидкостей частинок флюїда при малихта великих густинах. Пораховані радіальні функції розподілу ілюструють явище формування контактного шару на поверхні пор, яке приводить до зменшення рухливості молекул, адсорбованих в пористому середовищі. Отримані значення для коефіцієнта самодифузії добре узгоджуються із експериментальними даними для метану, адсорбованому в силікагелі.
\end{abstract}

Ключові слова: флюїд, рідина, пористе середовище, дифузія, комп'ютерне моделювання, молекулярна динаміка

PACS: $02.70 . N s, 45.20 . D d, 51.20 .+d, 61.43 . G t$ 
